BNL-113806-2017-JA

File \# 94762

\title{
Spline based least squares integration for two-dimensional shape or wavefront reconstruction
}

\author{
Lei Huang, Junpeng Xue, Bo Gao, Chao Zuo and Mourad Idir
}

Submitted to Optics and Lasers in Engineering

December 2, 2016

Photon Sciences Department

Brookhaven National Laboratory

\author{
U.S. Department of Energy \\ USDOE Office of Science (SC), \\ Basic Energy Sciences (BES) (SC-22)
}

Notice: This manuscript has been authored by employees of Brookhaven Science Associates, LLC under Contract No. DE- SC0012704 with the U.S. Department of Energy. The publisher by accepting the manuscript for publication acknowledges that the United States Government retains a non-exclusive, paid-up, irrevocable, world-wide license to publish or reproduce the published form of this manuscript, or allow others to do so, for United States Government purposes. 


\section{DISCLAIMER}

This report was prepared as an account of work sponsored by an agency of the United States Government. Neither the United States Government nor any agency thereof, nor any of their employees, nor any of their contractors, subcontractors, or their employees, makes any warranty, express or implied, or assumes any legal liability or responsibility for the accuracy, completeness, or any third party's use or the results of such use of any information, apparatus, product, or process disclosed, or represents that its use would not infringe privately owned rights. Reference herein to any specific commercial product, process, or service by trade name, trademark, manufacturer, or otherwise, does not necessarily constitute or imply its endorsement, recommendation, or favoring by the United States Government or any agency thereof or its contractors or subcontractors. The views and opinions of authors expressed herein do not necessarily state or reflect those of the United States Government or any agency thereof. 


\section{Spline based least squares integration}

\section{for two-dimensional shape or wavefront reconstruction}

\author{
Lei Huang, ${ }^{1, *}$ Junpeng Xue, ${ }^{1,2}$ Bo Gao, ${ }^{1,3,4}$ Chao zuo, ${ }^{5}$ and Mourad $\operatorname{Idir}^{1}$
}

1. Brookhaven National Laboratory - NSLS II, 50 Rutherford Dr. Upton, NY 11973-5000, USA

2. School of Aeronautics and Astronautics, Sichuan University, Chengdu 610065, China

3. Shanghai Institute of Applied Physics, Chinese Academy of Sciences, Shanghai 201800, China

4. University of Chinese Academy of Sciences, Beijing 100049, China

5. Jiangsu Key Laboratory of Spectral Imaging \& Intelligence Sense, Nanjing University of Science and Technology, Nanjing 210094, China

* Corresponding author: huanglei0114@gmail.com

Abstract: In this work, we present a novel method to handle two-dimensional shape or wavefront reconstruction from its slopes. The proposed integration method employs splines to fit the measured slope data with piecewise polynomials and uses the analytical polynomial functions to represent the height changes in a lateral spacing with the pre-determined spline coefficients. The linear least squares method is applied to estimate the height or wavefront as a final result. Numerical simulations verify that the proposed method has less algorithm errors than two other existing methods used for comparison. Especially at the boundaries, the proposed method has 
18 better performance. The noise influence is studied by adding white Gaussian noise to the slope data.

19 Experimental data from phase measuring deflectometry are tested to demonstrate the feasibility of

20 the new method in a practical measurement.

22 Keywords: shape reconstruction from gradient; wavefront reconstruction; splines;

\section{$24 \quad 1$ Introduction}

25 Two-dimensional integration methods [1-4] are widely applied to reconstruct the height or

26 wavefront from the measured gradient data in slope metrology, such as deflectometry [5, 6] and

27 wavefront sensing $[7,8]$ etc.

28 The pioneer studies in two-dimensional integration can be found in wavefront reconstruction since

29 the late 1970s [9-13]. Among these classical studies, Southwell's method [13] received great

30 attention and success because of its good performance and simple implementation with the

31 well-known Southwell geometry. It becomes the representative of the zonal integration methods.

32 However, the integration accuracy is limited since it assumes the height distribution between two

33 sampling points is only quadratic, which is not always true in reality. Based on this observation, an

34 iterative compensation method was proposed to improve the accuracy [14]. By analyzing the 
35 Taylor theorem and truncation error, Li et al. proposed a straightforward method with higher order

36 finite difference format [15], which is elegant and outperforms in a comparison [3] as it is more

37 accurate than the traditional Southwell's method, and faster than the iterative method. Recently,

38 Ren et al. proposed an easy implementation of Li's method for incomplete dataset or even in

39 arbitrary domain [16].

40 In this work, we present a novel spline based least squares method for two-dimensional shape or

41 wavefront reconstruction from slopes in rectangular grids. Benefitted from high accuracy of spline

42 fitting, the reconstruction accuracy can be improved. A comparative study with Southwell's

43 method and Li's method is conducted in this work. The three methods share the same grid

44 geometry (the Southwell geometry), as shown in Fig. 1. One of the beauties of this grid geometry is

45 that the height reconstruction happens exactly at the same locations of slope measurement.

\section{Principle}

47 In the proposed method, the zonal relations of the neighboring height values are described as

48

$$
\left\{\begin{array}{l}
z_{m, n+1}-z_{m, n}=\sum_{k=0}^{3} \frac{1}{k+1} c_{m, n, k}^{x} \Delta x_{m, n}^{k+1} \\
z_{m+1, n}-z_{m, n}=\sum_{k=0}^{3} \frac{1}{k+1} c_{m, n, k}^{y} \Delta y_{m, n}^{k+1}
\end{array},\right.
$$

49 where $\Delta x_{m, n}=x_{m, n+1}-x_{m, n}$ and $\Delta y_{m, n}=y_{m+1, n}-y_{m, n}$ are the $x$ - and $y$-step sizes at matrix location ( $m$,

$50 n$ ) as show in Fig. 1. $c_{m, n, k}^{x}$ and $c_{m, n, k}^{y}$ are the coefficients of the $k$ th order piecewise polynomials 
51 starting at $(m, n)$, which are determined through the cubic spline fitting of the $m$ th row of $x$-slopes

52 and the $n$th column of $y$-slopes, respectively.

64 boundary condition”.

65 By integrating the analytical polynomial functions in Eq. (2) or Eq. (3) with the spline determined

66 coefficients $c_{m, n, k}^{x}$ or $c_{m, n, k}^{y}$, the height difference after a lateral step $\Delta x_{m, n}$ or $\Delta y_{m, n}$ can be 
67 calculated through the right hand sides of Eq. (1). The linear least squares solution of height can be

68 described as

69

$$
\left[\begin{array}{c}
z_{1,1} \\
z_{2,1} \\
\vdots \\
z_{M, N}
\end{array}\right]=\left(\mathbf{D}^{\mathrm{T}} \mathbf{D}\right)^{-1} \mathbf{D}^{\mathrm{T}} \mathbf{G},
$$

70 where the symbol $(\cdot)^{\mathrm{T}}$ stands for the transpose operation, and $(\cdot)^{-1}$ is the matrix inverse. The

71 sparse matrix $\mathbf{D}$ and vector $\mathbf{G}$ are

72

$$
\mathbf{D}=\left[\begin{array}{ccccccccc}
-1 & 0 & \cdots & 0 & 1 & 0 & \cdots & \cdots & 0 \\
0 & -1 & 0 & \cdots & 0 & 1 & 0 & \cdots & 0 \\
\vdots & \vdots & \vdots & \vdots & \vdots & \vdots & \vdots & \vdots & \vdots \\
0 & \cdots & \cdots & 0 & -1 & 0 & \cdots & 0 & 1 \\
-1 & 1 & 0 & \cdots & \cdots & \cdots & \cdots & \cdots & 0 \\
0 & -1 & 1 & 0 & \cdots & \cdots & \cdots & \cdots & 0 \\
\vdots & \vdots & \vdots & \vdots & \vdots & \vdots & \vdots & \vdots & \vdots \\
0 & \cdots & \cdots & \cdots & \cdots & \cdots & 0 & -1 & 1
\end{array}\right] .
$$

73

$$
\mathbf{G}=\left[\begin{array}{c}
\sum_{k=0}^{3} \frac{1}{k+1} c_{k, 1,1}^{x} \Delta x_{1,1}^{k+1} \\
\sum_{k=0}^{3} \frac{1}{k+1} c_{k, 2,1}^{x} \Delta x_{2,1}^{k+1} \\
\vdots \\
\sum_{k=0}^{3} \frac{1}{k+1} c_{k, M, N-1}^{x} \Delta x_{M, N-1}^{k+1} \\
\sum_{k=0}^{3} \frac{1}{k+1} c_{k, 1,1}^{y} \Delta y_{1,1}^{k+1} \\
\sum_{k=0}^{3} \frac{1}{k+1} c_{k, 2,1}^{y} \Delta y_{2,1}^{k+1} \\
\vdots \\
\sum_{k=0}^{3} \frac{1}{k+1} c_{k, M-1, N}^{y} \Delta y_{M-1, N}^{k+1}
\end{array}\right] .
$$




\section{$74 \quad 3 \quad$ Simulation}

75 In order to illustrate the excellent performance of the proposed method, a two-dimensional cosine

76 function with varying local frequencies $z=\cos \left(2 \pi x^{2} / 3000\right) \cdot \cos \left(2 \pi y^{2} / 3000\right)$ is selected as the

77 Surface Under Test (SUT) to reconstruct as shown in Fig. 2(a). Its corresponding analytically

78 derived $x$-slope and $y$-slope are shown in Fig. 2(b) and Fig. 2(c). We set $x$-unit the same as $y$-unit

79 and named as "lateral unit,” [l. u.], and z-unit is symbolled as [z. u.]. The in-plane coordinates are

80 sampled as $x=1,2 \ldots, 256$ [l.u.] and $y=1,2 \ldots, 256$ [l.u.]. The value of height ranges in \pm 1 [z. u.],

81 and both $x$-slope and $y$-slope range within \pm 1 [z. u. / l. u.], so the Peak-To-Valley (PTV) of the

82 slopes are $2[$ z. u. / l. u.] for the simulated SUT.

91 [15]. It is a big advantage in handling huge slope datasets. This is one of reasons why we compare

Fig. 2. A surface height (a) with varying local frequencies is chosen as the benchmark in simulation to test the performance of different methods in height reconstruction from $x$-slope (b), and

$$
y \text {-slope(c). }
$$

Three integration methods (Southwell's method [13], Li's algorithm 1 in Ref. [15], and our spline-based method in this work) are applied to reconstruct height from the slopes in Fig. 2(b-c)

for a comparison. All these methods share the same sparse matrix $\mathbf{D}$, which has the less memory cost and computing time for the matrix inverse operation comparing to other two algorithms in Ref. these three methods.
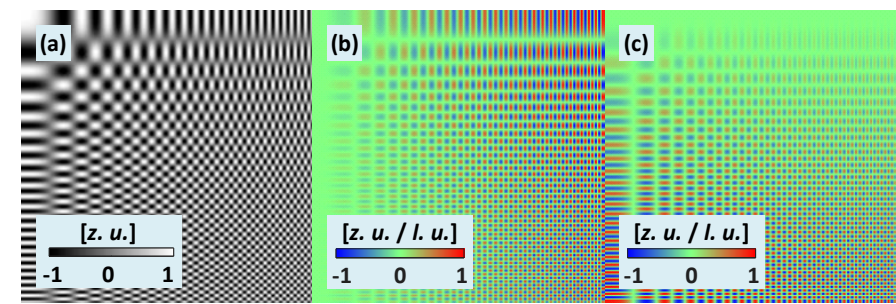
93 The reconstruction errors are illustrated in Fig. 3. It indicates that these zonal methods make larger

94 reconstruction errors in higher frequency regions. For comparison purposes, Southwell's method

95 has the largest reconstruction error with its Root Mean Square $(\mathrm{RMS})=2.6 \times 10^{-2}[z . u$. $]$ and PTV $=$

960.19 [z. u.]. Li's reconstruction method ends up with errors of RMS $=5.8 \times 10^{-3}[z . u$. $]$ and PTV $=$

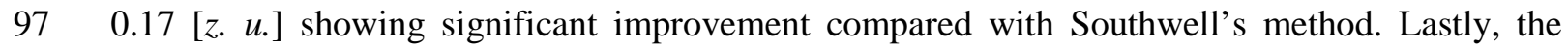

98 proposed method outperforms the others with reconstruction errors of RMS $=9.6 \times 10^{-4}[z$. u.] and

$99 \quad \mathrm{PTV}=0.03[z . u$. $]$ only.

100

101

102

103

104

105

106

107

108

109
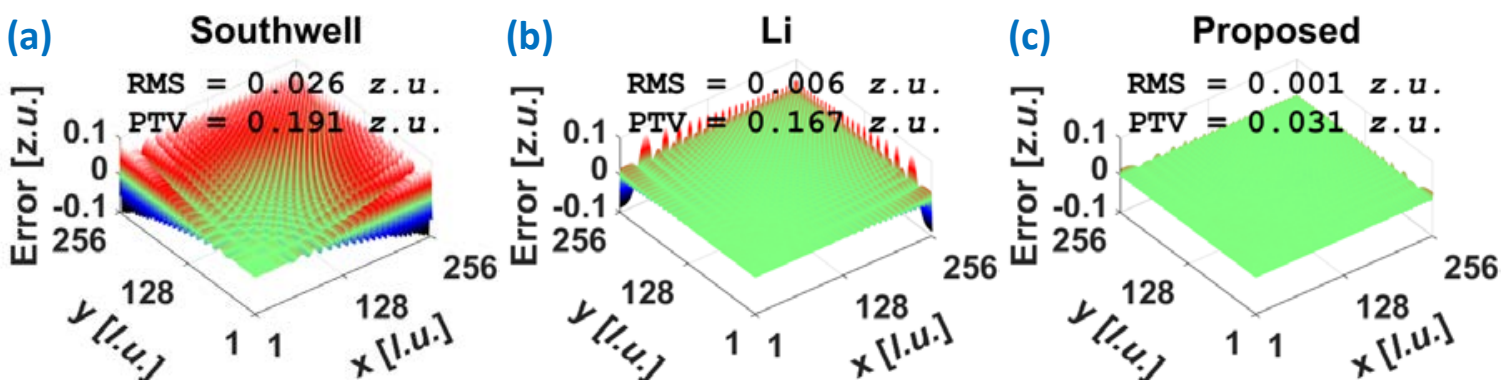

Fig. 3. The proposed method (c) has less errors comparing to the two existing methods: (a) Southwell's method, (b) Li's method. The codes in MATLAB ${ }^{\circledR}$ can be found in [17].

It is obvious that the proposed spline-based method has better estimation at regions with high-frequency variations. More significantly, splines have naturally good performance at dataset boundaries. In contrast, four neighboring slopes in one direction are always required in Li's algorithm 1 in Ref. [15] which cannot be satisfied at the boundaries.

Here we set the measuring range of the slope sensor as 5 times the slope PTV of the SUT in our simulation, which is a reasonable number in practical measurements. The simulated slopes PTV is $2[$ z. u. / l. u.], so the slope measuring range is set as $10[z$. u. / l. u.] here. We define the 
110 Range-to-Precision Ratio (RPR) as the ratio of the slope measuring range of the sensor over its

111 slope measuring precision. The RPR varies from $10^{2}$ up to $10^{5}$, which is a typical range for slope

112 sensors in various applications. Additive white Gaussian noises with different standard deviations

113 according to different RPR values are simulated into slopes to study the behavior of integrators

114 under noise conditions.

115

116

117

118

119

120

121 These three methods offer very similar results when the RPR is only $10^{2}$. When the RPR is

122 approaching $10^{3}$, the noise still dominates the total reconstruction error, but it can be noticed that

Fig. 4. While the RPR of the slope sensor increases (slope noise decreasing with a constant measuring range in our simulation) from $10^{2}$ to $10^{5}$, the error owing to the slope noise dominates the reconstruction error at first and then gradually has less impact comparing to the truncation error of algorithms. When RPR is higher than $10^{4}$, the reconstruction accuracy of the proposed method is much better than the other methods in comparison, especially in high frequency regions.

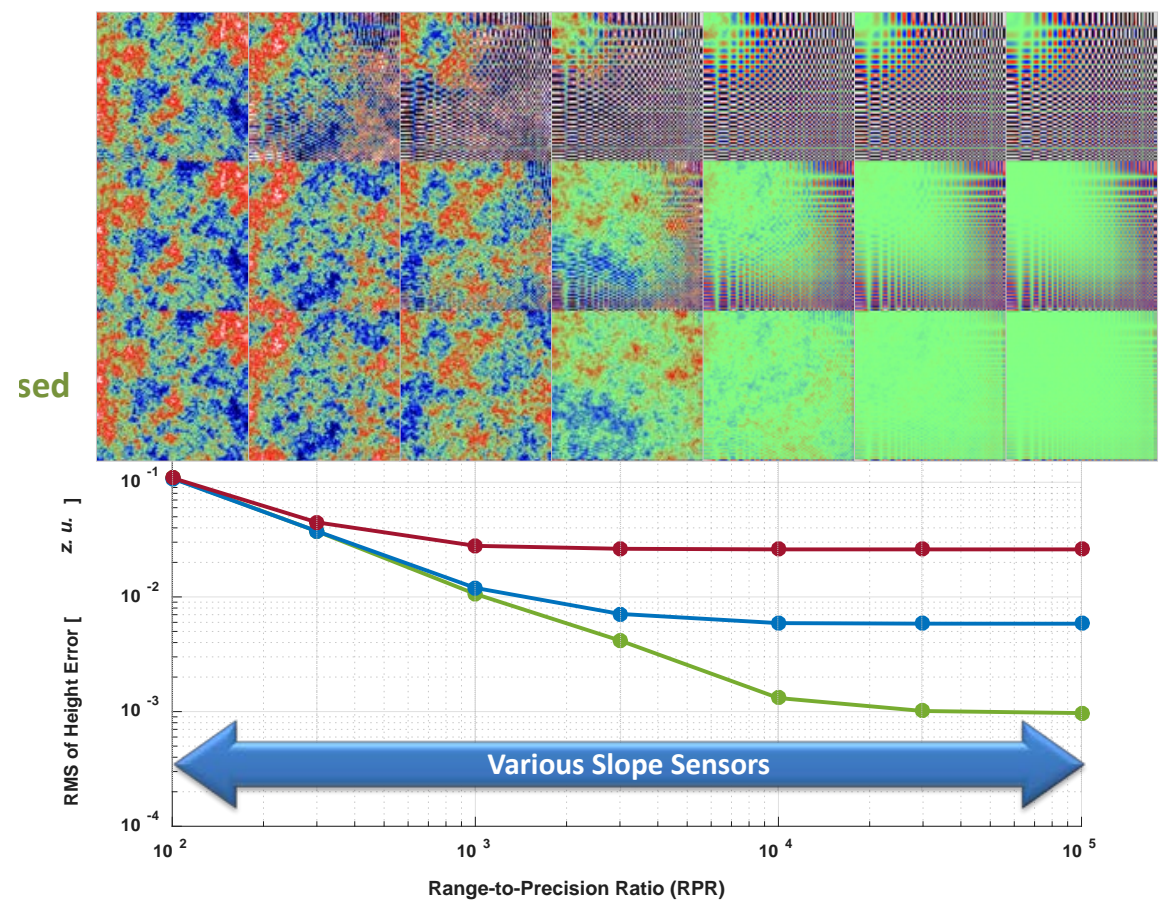

approaching 10, the noise still dominates the total reconstruction enror, but it can be noticed that

123 the errors from Li's method and the proposed method are getting smaller than Southwell's result.

124 When the RPR is higher than $10^{4}$, the truncation error in numerical calculation especially in the Page 8 of 15 
125 high frequency region makes the major contribution to the total error for Southwell's and Li's 126 methods. The proposed spline-based method performs better comparing to the other two methods

127 in this range. It indicates an accurate integration method becomes more important when measuring

128 high-frequency surface variations by using a higher precision slope metrology tool, such as the 129 stitching shack-Hartmann optical head [18] which typically has a RPR value about $10^{5}$.

130

131

132

133

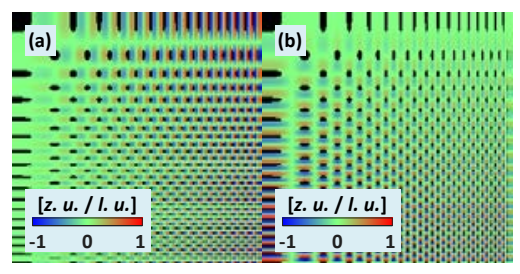

Fig. 5. Incomplete $x$-slope (a) and $y$-slope (b) data are simulated to study the performance of the proposed method.

Moreover, the proposed method can be used to handle incomplete slope datasets. In this condition, the spline fitting is implemented section by section instead of an entire vector, due to the segmentation by invalid regions with no slope available. At the boundaries of the invalid region, the cubic spline has its good natural solution once at least 4 valid pixels are connected in one direction. If there are only 3 or 2 slope values in one section, a lower order spline will be used in fitting those slopes or Southwell-derived G values can become a backup. 

$152\left(5 \times 10^{-3}\right.$ z. u. RMS and $5.7 \times 10^{-2}$ z. u. PTV) in Fig. 6(d). their difference. (a)

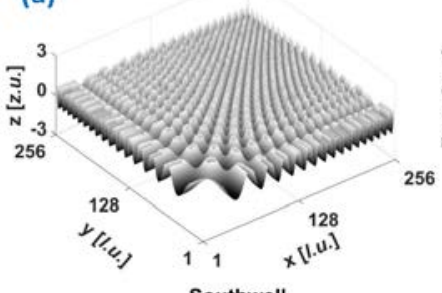

(c)

Southwell

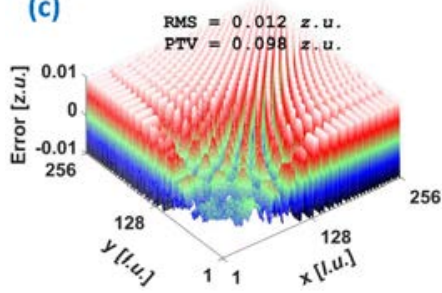

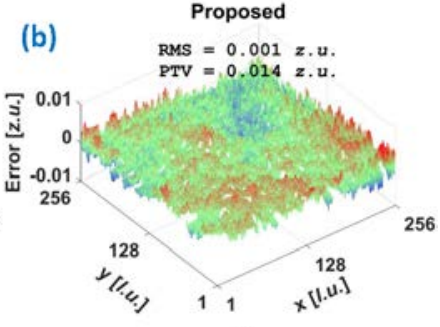

Li

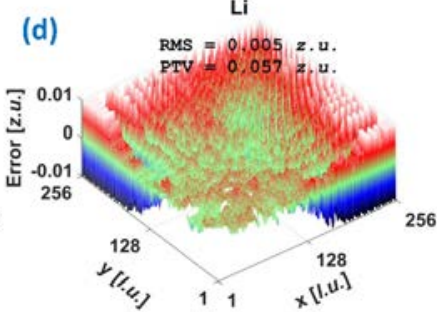

Fig. 6. The SUT can be reconstructed by the proposed method even if there are invalid slope regions (a). The error of the proposed reconstruction (b) is smaller than those of the Southwell's method (c) and Li's method (d). The same axes limits and color map are set in (b)-(d) to highlight

As illustrated in Fig. 5, some of the slope values are removed from the slopes in Fig. 2, which is common in real measurements due to the unacceptable surface quality. The performance of the proposed spline-based integration method is tested with this type of incomplete slope dataset. The RPR of the measurement system is set as $10^{4}$ to have certain slope noise in the simulation. The proposed method can accurately reconstruct the surface as shown in Fig. 6(a). Of course, the regions with no slope data cannot be reconstructed in principle. The reconstruction error in Fig. 6(b) is only $1 \times 10^{-3}$ z. u. RMS and $1.4 \times 10^{-2}$ z. u. PTV, comparing to the reconstruction errors of Southwell's method $\left(1.2 \times 10^{-2}\right.$ z. u. RMS and $9.8 \times 10^{-2}$ z. u. PTV) in Fig. 6(c) and Li's method

\section{Experiment}


154 To demonstrate the feasibility of the proposed method in an actual measurement, experimental

155 slope results from phase measuring deflectometry are used as a data sample for reconstruction. The

156 SUT is about $15 \mathrm{~mm} \times 15 \mathrm{~mm}$ with slopes ranging in \pm 17 mrad. The slopes are integrated by the

157 proposed method as illustrated in Fig. 7.

158

159

160

161
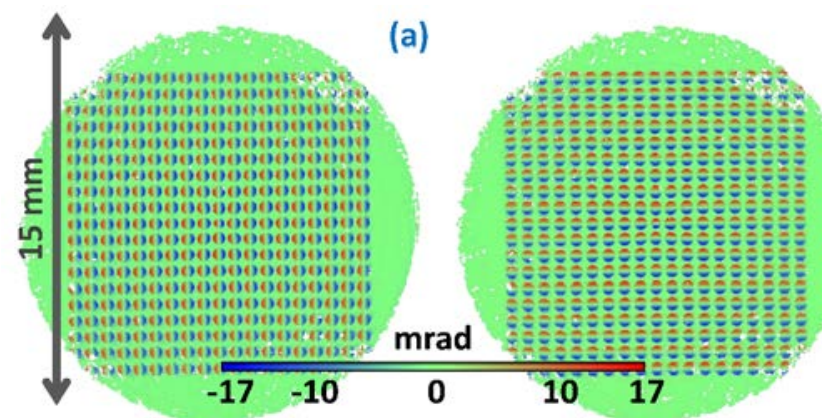

(b)
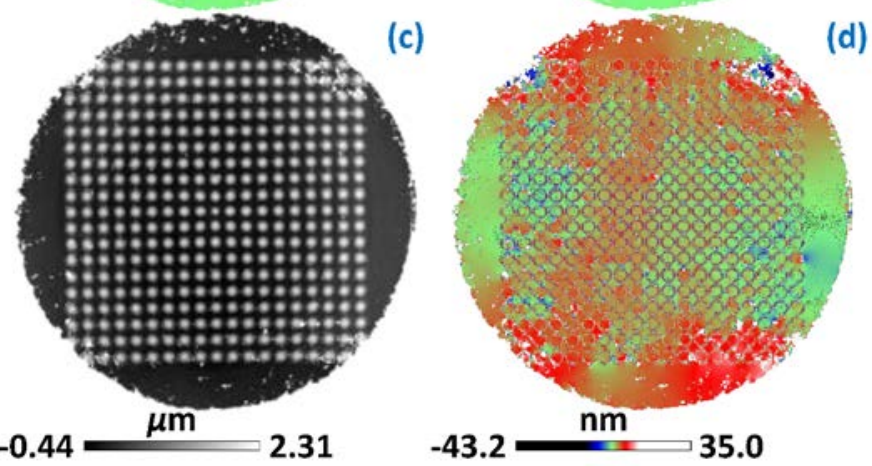

(d)

Fig. 7. Experimental $x$-slope (a) and $y$-slope (b) from deflectometry are integrated by using the proposed spline-based method, and the resultant height map (c) has a reconstruction difference (d) comparing to Li's method.

Although there are many invalid data in the measured slopes and the aperture shape is not rectangular, the proposed method still can successfully reconstruct the surface height as shown in

164 Fig. 7(c). The difference of two reconstructions (the proposed method vs. Li's method) is illustrated

165 in Fig. 7(d). The differences around the edges of the bumps on the surface are relatively bigger due

166 to the rapid changes in slopes locally. 


\section{Discussion}

168 Since the spline fitting of slopes takes additional time, the speed of the proposed spline-based

169 integration method is slower than the other two zonal methods, but their speed difference is

170 insignificant. Fig. 8 compares the time of using these three methods for different slope data size.

171 Generally speaking, the proposed method takes 30\%-80\% longer time than Li's method does

172 depending on the data size. The larger size yields the lower ratio of their speed difference.

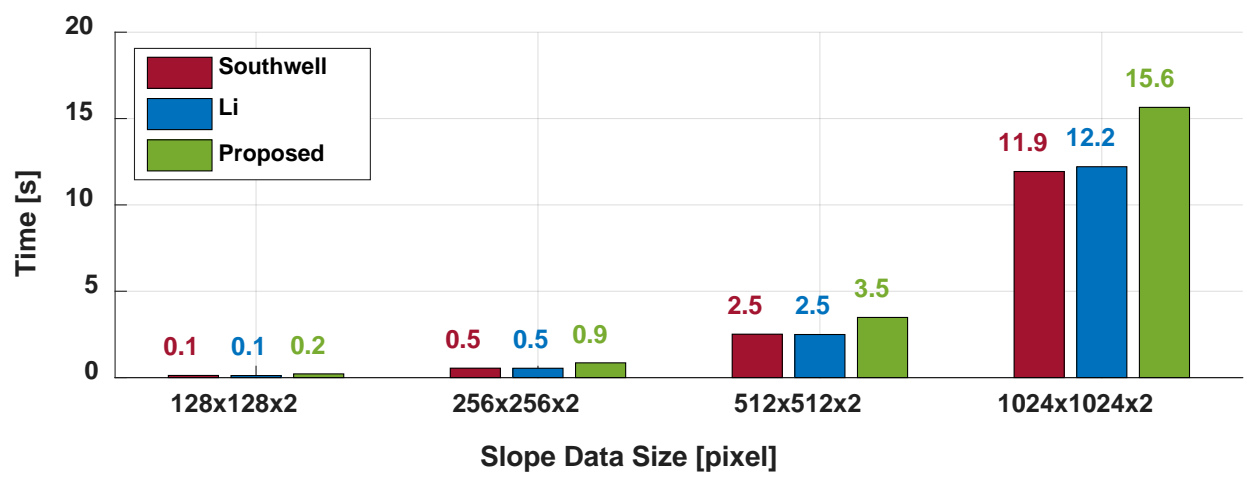

173

174

175

The new method is proposed to deal with slope data in rectangular grids, and it has an intrinsic

limitation to process the slopes in quadrilateral grids, or the even more general triangular grids.

178 This can be a potential work for future.

\section{Conclusion}

180 In summary, we present a novel two-dimensional integration method in this work to reconstruct the

181 height or wavefront from its sampled first derivatives (the measured slopes). The well-established 
182 spline technique is employed to fit the slopes with piecewise polynomials. The analytical

183 polynomial functions with determined coefficients are employed to calculate the height variations

184 for each spatial step in $x$-and $y$-directions. The final height result is estimated with the linear least

185 squares method. The codes in $\mathrm{MATLAB}^{\circledR}$ can be downloaded from [17]. A comparison with two

186 similar existing integration techniques and the noise influence are investigated through simulation

187 to reveal the advantages of the proposed method. The proposed method show great performance at

188 the boundaries of the dataset or holes. Working with the experimental deflectometry slope data

189 demonstrates the proposed method is feasible and effective in a practical integration.

\section{$190 \quad$ Funding}

\section{Acknowledgement}

193 We are thankful to Josep Nicolas (ALBA-SLAC) for the helpful discussions.

\section{References:}

195 [1] M. Ares, S. Royo, Comparison of cubic B-spline and Zernike-fitting techniques in complex 196 wavefront reconstruction, Appl. Opt., 45 (2006) 6954-6964.

197 [2] L. Huang, A.K. Asundi, Framework for gradient integration by combining radial basis 198 functions method and least-squares method, Appl. Opt., 52 (2013) 6016-6021. 
199 [3] L. Huang, M. Idir, C. Zuo, K. Kaznatcheev, L. Zhou, A. Asundi, Comparison of 200 two-dimensional integration methods for shape reconstruction from gradient data, Optics and 201 Lasers in Engineering, 64 (2015) 1-11.

202 [4] F. Dai, F. Tang, X. Wang, O. Sasaki, P. Feng, Modal wavefront reconstruction based on Zernike 203 polynomials for lateral shearing interferometry: comparisons of existing algorithms, Appl. Opt., 51 204 (2012) 5028-5037.

205 [5] S. Ettl, J. Kaminski, M.C. Knauer, G. Häusler, Shape reconstruction from gradient data, Appl. 206 Opt., 47 (2008) 2091-2097.

207 [6] H. Ren, F. Gao, X. Jiang, Least-squares method for data reconstruction from gradient data in 208 deflectometry, Appl. Opt., 55 (2016) 6052-6059.

209 [7] I. Mochi, K.A. Goldberg, Modal wavefront reconstruction from its gradient, Appl. Opt., 54 210 (2015) 3780-3785.

211 [8] L. Seifert, H.J. Tiziani, W. Osten, Wavefront reconstruction with the adaptive Shack-Hartmann 212 sensor, Optics Communications, 245 (2005) 255-269.

213 [9] D.L. Fried, Least-square fitting a wave-front distortion estimate to an array of phase-difference 214 measurements, J. Opt. Soc. Am., 67 (1977) 370-375.

215 [10] R.H. Hudgin, Wave-front reconstruction for compensated imaging, J. Opt. Soc. Am., 67 $216 \quad$ (1977) 375-378.

217 [11] R.H. Hudgin, Optimal wave-front estimation, J. Opt. Soc. Am., 67 (1977) 378-382.

218 [12] R.J. Noll, Phase estimates from slope-type wave-front sensors, J. Opt. Soc. Am., 68 (1978) 219 139-140.

220 [13] W.H. Southwell, Wave-front estimation from wave-front slope measurements, J. Opt. Soc. 221 Am., 70 (1980) 998-1006.

222 [14] L. Huang, A. Asundi, Improvement of least-squares integration method with iterative 223 compensations in fringe reflectometry, Appl. Opt., 51 (2012) 7459-7465.

224 [15] G. Li, Y. Li, K. Liu, X. Ma, H. Wang, Improving wavefront reconstruction accuracy by using 225 integration equations with higher-order truncation errors in the Southwell geometry, J. Opt. Soc. 226 Am. A, 30 (2013) 1448-1459. 
227 [16] H. Ren, F. Gao, X. Jiang, Improvement of high-order least-squares integration method for 228 stereo deflectometry, Appl. Opt., 54 (2015) 10249-10255.

229 [17] L. Huang, MATLAB codes for spline based least squares integration for two dimensional 230 shape or wavefront reconstruction, 231 https://github.com/huanglei0114/Spline-based-least-squares-integration-for-two-dimensional-sha 232 pe-or-wavefront-reconstruction.

233 [18] M. Idir, K. Kaznatcheev, G. Dovillaire, J. Legrand, R. Rungsawang, A 2 D high accuracy 234 slope measuring system based on a Stitching Shack Hartmann Optical Head, Opt. Express, 22 235 (2014) 2770-2781. 